\title{
Clinical, Biochemical, Hematological, Endocrinological and Immunological Differences Between Graves' Disease Patients With and Without Thyroid Storm
}

\author{
Hidekatsu Yanai $^{\mathrm{a}, \mathrm{b}}$, Mariko Hakoshima ${ }^{\mathrm{a}}$, Hisayuki Katsuyama ${ }^{\mathrm{a}}$
}

\begin{abstract}
Background: Thyroid storm (TS) is very rare, however, a life-threatening medical condition requiring emergency treatment. Since TS is rare, published case reports and seven unpublished cases of TS diagnosed at the researchers' facilities were analyzed to make diagnostic criteria for TS in Japan. There are no reports on differences in backgrounds between Graves' disease patients with and without TS, from a single research facility.
\end{abstract}

Methods: We retrospectively picked up patients who had been diagnosed as having Graves' disease with and without TS, at National Center for Global Health and Medicine Kohnodai Hospital, between January 2010 and October 2018. According to the guideline for the diagnosis of Graves' disease and TS presented by the Japan Thyroid Association, we diagnosed patients as having Graves' disease with and without TS. We obtained clinical and laboratory data by using electronical medical records and database after showing the opt-out.

Results: We found 69 Graves' disease patients without TS, and five Graves' disease patients with TS. Graves' disease patients with TS were significantly younger than those without TS. Graves' disease patients with TS included a significantly higher percentage of male patients than those without TS. Body temperature and pulse rate in patients with TS were significantly higher than those without TS. Serum thyroid hormone levels and the titer of third-generation thyroid stimulating hormone receptor antibody in patients with TS were significantly higher than in those without TS. Neutrophilic leukocytosis together with eosinopenia, monocytopenia and lymphocytopenia were observed in patients with TS. Serum alkaline phosphatase level was significantly higher in patients with TS than in those without TS. Serum levels of triglyceride, high-density lipoprotein-cholesterol, and low-density lipoprotein-cholesterol in patients with TS were sig-

Manuscript submitted April 8, 2019, accepted April 22, 2019

a Department of Internal Medicine, National Center for Global Health and Medicine Kohnodai Hospital, Chiba, Japan

${ }^{\mathrm{b}}$ Corresponding Author: Hidekatsu Yanai, Department of Internal Medicine, National Center for Global Health and Medicine Kohnodai Hospital, 1-7-1 Kohnodai, Ichikawa, Chiba 272-8516, Japan.

Email: dyanai@hospk.ncgm.go.jp

doi: https://doi.org/10.14740/jocmr3833 nificantly lower than those without TS.

Conclusions: Our study demonstrated significant clinical, biochemical, hematological, endocrinological and immunological differences in Graves' disease patients with TS compared to those without TS.

Keywords: Graves' disease; White blood cells; Thyroid storm; TSH receptor antibody; Serum lipids

\section{Introduction}

Thyroid storm (TS) is very rare, however, a life-threatening medical condition requiring emergency treatment [1-3]. Nationwide surveys were conducted from 2004 through 2008 to investigate TS incidence in Japan $[3,4]$. The incidence rate was estimated to be 0.20 persons per 100,000 population per year in Japan, accounting for $0.22 \%$ of all thyrotoxic patients and $5.4 \%$ of hospitalized thyrotoxic patients. The mortality rate of patients with definite or suspected TS was $10.7 \%$. Individually, the mortality rates for definite and suspected cases were $11.0 \%$ and $9.5 \%$, respectively [3]. The most common causes of death in patients with TS were multiple organ failure (MOF) and congestive heart failure (CHF) $[3,5]$, reflecting the decompensation of multiple organs due to thyrotoxicosis.

Since TS is rare, a prospective study that develops criteria was considered very difficult and time-consuming. Therefore, the criteria were mainly based on information obtained from the literatures [6]. Ninety-three original case reports and seven unpublished cases of TS diagnosed at the researchers' facilities were collected, and clinical parameters extracted from these cases were analyzed [6]. Based on the analysis of these data, the first edition of diagnostic criteria for TS was created, and its incidence in Japan was surveyed [3]. By such survey, "definite" TS was defined as thyrotoxicosis plus at least one central nervous system (CNS) manifestation and one of the followings: fever, tachycardia, CHF, or gastrointestinal (GI)/ hepatic manifestation; thyrotoxicosis and at least three of the followings: fever, tachycardia, CHF, or GI/hepatic manifestations [7].

We retrospectively studied clinical, biochemical, hematological, endocrinological and immunological differences 
Table 1. Clinical Differences Between Graves' Disease Patients With and Without TS

\begin{tabular}{|c|c|c|c|c|c|}
\hline & \multicolumn{2}{|c|}{ Graves' disease without TS } & \multicolumn{2}{|c|}{ Graves' disease with TS } & \multirow{2}{*}{ P value } \\
\hline & $\mathbf{N}$ & Value & $\mathbf{N}$ & Value & \\
\hline Age (years) & 69 & $49.0 \pm 18.0$ & 5 & $38.4 \pm 8.3$ & 0.042 \\
\hline Sex (female, n (\%)) & 69 & $55(79.7 \%)$ & 5 & $3(60 \%)$ & $<0.001$ \\
\hline Body temperature $\left({ }^{\circ} \mathrm{C}\right)$ & 35 & $36.7 \pm 0.4$ & 5 & $38.5 \pm 0.9$ & 0.012 \\
\hline
\end{tabular}

Values except for sex indicate mean \pm SD. TS: thyroid storm.

between Graves' disease patients with and without TS whose diagnosis were done in the National Center for Global Health and Medicine Kohnodai Hospital, a single research facility, in Japan.

\section{Materials and Methods}

\section{Study population}

We retrospectively picked up patients who had been diagnosed as having Graves' disease with and without TS, at National Center for Global Health and Medicine Kohnodai Hospital, between January 2010 and October 2018. According to the guideline for the diagnosis of Graves' disease presented by the Japan Thyroid Association, we diagnosed patients as having Graves' disease by observing signs of thyrotoxicosis, elevation in serum free thyroxine (fT4) and free triiodothyronine (fT3) levels, suppression of serum thyroid stimulating hormone (TSH) and positive for anti-TSH receptor antibody (TRAb). Such a patient shall be said probably to have Graves' disease by this guideline. TRAb levels had been measured by using the first, second, and third-generation TRAb measurement methods between January 2010 and October 2018, in our hospital. Further, according to the diagnostic criteria for TS of the Japan Thyroid Association [7], we diagnosed patients as having TS. In this guideline, presence of thyrotoxicosis with elevated levels of fT3 or fT4 is needed as the prerequisite for diagnosis. Thyrotoxicosis and at least one CNS manifestation and fever, tachycardia, $\mathrm{CHF}$, or GI/hepatic manifestations, and thyrotoxicosis and at least three combinations of fever, tachycardia, $\mathrm{CHF}$, or $\mathrm{GI} /$ hepatic manifestations are required to diagnose "definite" TS [7]. We obtained clinical and laboratory data by using electronical medical records and database after showing the opt-out.

\section{Statistical analysis}

Statistical analyses were performed by using SPSS version 23 (IBM Co., Ltd., Chicago, IL). All values are expressed as the mean \pm standard deviation except for sex. We performed the $t$-test in the comparison between variables between Graves' disease patients with and without TS. P value of $<0.05$ was considered statistically significant.

\section{Ethics statements}

Because this study was a retrospective cross-sectional observational study, the opt-out method was adopted. The study protocol was approved by the Ethics Committee of the National Center for Global Health and Medicine (NCGMG-003120-00), and the study was performed in accordance with the Declaration of Helsinki.

\section{Results}

We found 69 Graves' disease patients without TS, and five Graves' disease patients with TS.

\section{Clinical differences between Graves' disease patients with and without TS}

Clinical differences between Graves' disease patients with and without TS were shown in Table 1. Graves' disease patients with TS were significantly younger than those without TS. Graves' disease patients with TS included a significantly higher percentage of male patients than those without TS. Body temperature and pulse rate in patients with TS were significantly higher than those without TS.

Differences in TSH, thyroid hormones and thyroid autoantibodies between Graves' disease patients with and without TS

Differences in TSH, thyroid hormones and thyroid autoantibodies between Graves' disease patients with and without TS were shown in Table 2. There were no significant differences in TSH and the ratio of fT3 to fT4 between Graves' disease patients with and without TS. Serum fT3 and fT4 levels in patients with TS were significantly higher than in those without TS. The titer of TRAb in all five patients had been measured by the third-generation TRAb measurement method. All patients showed the titer of third-generation $\mathrm{TRAb}>30 \mathrm{IU} / \mathrm{L}$. To statistically analyze the difference in the titer of third-generation TRAb between patients with and without TS, the titer of patients with TS was assumed to be $30 \mathrm{IU} / \mathrm{L}$. The titer of the third-generation TRAb in Graves' 
Table 2. Differences in TSH, Thyroid Hormones and Thyroid Autoantibodies Between Graves' Disease Patients With and Without TS

\begin{tabular}{|c|c|c|c|c|c|}
\hline & & disease without TS & & es' disease with TS & P value \\
\hline & $\mathbf{N}$ & Values & $\mathbf{N}$ & Values & \\
\hline $\mathrm{TSH}(\mu \mathrm{IU} / \mathrm{mL})$ & 69 & $0.03 \pm 0.01$ & 5 & $0.03 \pm 0.00$ & 0.253 \\
\hline Free T4 $(\mu \mathrm{g} / \mathrm{dL})$ & 69 & $3.7 \pm 1.9$ & 5 & $7.1 \pm 1.8$ & 0.011 \\
\hline Free T3/Free T4 & 61 & $3.6 \pm 0.7$ & 5 & $3.4 \pm 0.5$ & 0.481 \\
\hline TPO-Ab (IU/mL) & 53 & $151.6 \pm 199.2$ & 4 & $464.0 \pm 272.0$ & 0.103 \\
\hline Tg-Ab (IU/mL) & 51 & $207.4 \pm 257.3$ & 3 & $1,863.0 \pm 1,688.8$ & 0.231 \\
\hline
\end{tabular}

Values indicate mean \pm SD. TS: thyroid storm; Tg-Ab: thyroglobulin antibody; TPO-Ab: thyroid peroxidase antibody; TSH: thyroid-stimulating hormone; T3: triiodothyronine; T4: thyroxine; TRAb: TSH receptor antibody.

disease patients with TS was significantly higher than those without TS.

\section{Differences in white blood cell (WBC) fractions between Graves' disease patients with and without TS}

Differences in WBC fractions between Graves' disease patients with and without TS were shown in Table 3. There was no significant difference in WBC counts between Graves' disease patients with and without TS. However, percentage of neutrophil among WBC was significantly higher in Graves' disease patients with TS than in those without TS. Furthermore, the percentages of lymphocyte, monocyte and eosinophil in patients with TS were significantly lower than in those without TS.

\section{Biochemical differences between Graves' disease patients with and without TS}

Biochemical differences between Graves' disease patients with and without TS were shown in Table 4. Serum alkaline phosphatase (ALP) level was significantly higher in Graves' disease patients with TS than in those without TS. Serum levels of triglyceride (TG), high-density lipoprotein-cholesterol (HDL-C), and low-density lipoprotein-cholesterol (LDL-C) in patients with TS were significantly lower than those without TS.

\section{Discussion}

Because TS is too rare, prospective studies have been considered very difficult and time-consuming to make diagnostic criteria for TS. Therefore, ninety-three published original case reports and seven unpublished cases of TS diagnosed at the researchers' facilities were used to make diagnostic criteria for TS [6]. The present diagnostic criteria are based on thyrotoxicosis and physical findings such as CNS manifestation, tachycardia and fever [7]. Early suspicion and diagnosis, and prompt and intensive treatment may improve survival of TS patients. Understanding of objective characteristics detected by laboratory examinations for TS may lead to early and accurate diagnosis of TS. Therefore, we retrospectively picked up Graves' disease patients with and without TS whose diagnosis were done in our hospital, a single research facility, and we studied clinical, biochemical, hematological, endocrinological and immunological differences between Graves' disease patients with and without TS.

In the previous study, both age (42.1 years old) in Graves' disease patients with TS reported in the literature and age (44.7 years old) in Graves' disease patients with TS in the survey of Japanese patients were similar with age (43.2 years old) in patients without TS [3]. However, our patients with TS (38.4 years old) were significantly younger than those without TS (49.0 years old). Both the frequency of female $(71.7 \%)$ in patients with TS reported in the literature and the frequency (73.4\%) in patients with TS in the survey of Japanese patients

Table 3. Differences in White Blood Cell Fractions Between Graves' Disease Patients With and Without TS

\begin{tabular}{|c|c|c|c|c|c|}
\hline & & ves' disease without TS & & aves' disease with TS & \\
\hline & $\mathbf{N}$ & Value & $\mathbf{N}$ & Value & Panue \\
\hline White blood cells $(/ \mathrm{mL})$ & 66 & $5,527.3 \pm 1,780.4$ & 5 & $7,480.0 \pm 5622.5$ & 0.482 \\
\hline Lymphocytes (\%) & 56 & $36.7 \pm 10.8$ & 5 & $16.1 \pm 10.5$ & 0.009 \\
\hline Monocytes (\%) & 56 & $7.7 \pm 2.5$ & 5 & $5.3 \pm 1.9$ & 0.044 \\
\hline
\end{tabular}

Values indicate mean \pm SD. TS: thyroid storm. 
Table 4. Biochemical Differences Between Graves' Disease Patients With and Without TS

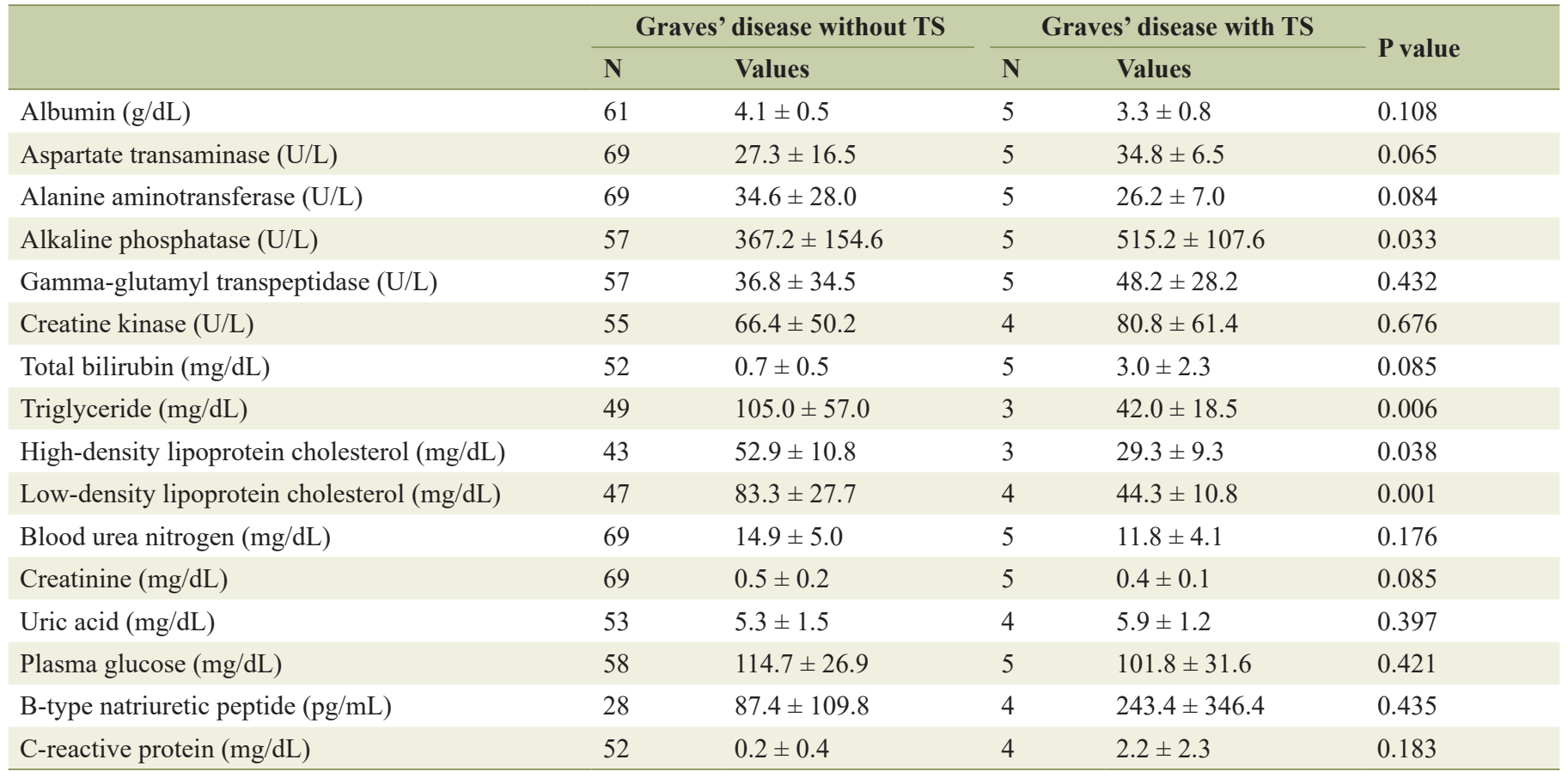

Values indicate mean \pm SD. TS: thyroid storm.

were similar with those $(74.4 \%)$ in patients without TS [3]. However, our study showed that the frequency of female $(60 \%)$ in patients with TS was significantly lower than that $(79.7 \%)$ in patients without TS. There have been no other reports on these data due to the rarity of TS, and further studies are required in the future. Body temperature and pulse rate were significantly higher in TS patients because TS was diagnosed according to the diagnostic criteria of the Japanese Thyroid Association [7].

In the previous study, serum fT4 and fT3 concentrations were similar among patients with TS in the literature, Japanese patients with "definite" TS or "suspected" TS, and a group of patients with thyrotoxicosis without TS [3]. However, our study showed that serum fT3 and fT4 levels in patients with TS were significantly higher than in those without TS. There have been also no other reports on these data due to the rarity of TS, and further studies are desired to confirm this.

To our knowledge, our study is the first to show neutrophilic leukocytosis together with eosinopenia, monocytopenia and lymphocytopenia in TS. Corticosteroids are considered essential stress hormones. Steroid levels increase immediately after stress such as injury, pain, fever and hypovolemia in response to the stimulation of corticotropin-releasing hormone secretion by various cytokines [8]. The increase in steroid levels is typically proportional to the magnitude of stress, with serum cortisol values being highest in moribund patients and shortly before death [8]. The elevation of steroid may be remarkably higher in patients with TS than those without TS. Steroid has been reported to cause neutrophilic leukocytosis together with eosinopenia, monocytopenia, and lymphocytopenia [9]. Neutrophilic leukocytosis, eosinopenia, monocytopenia, and lymphocytopenia which were observed in our study may be induced by excess steroid release due to intense stress such as TS. Four of five TS patients showed the absence of eosinophil ( $0 \%$ ) and one TS patient showed $1 \%$ of eosinophil, suggesting that eosinopenia is a useful marker for TS.

Serum ALP level in TS patients was significantly higher than patients without TS. This may reflect an increased bone and mineral metabolism due to hyperthyroidism $[10,11]$. Otherwise, this may reflect GI/hepatic manifestation in TS, which was supported by non-significantly elevated total bilirubin levels in TS [3]. Serum levels of TG, HDL-C and LDL-C levels were significantly and remarkably lower in TS patients than patients without TS. In a multiple logistic regression analysis of previous study [3], serum total cholesterol level was significantly related with the severity and prognosis of TS, which was consistent with our result. However, the underlying mechanisms for severe hypolipidemia in TS remain completely unknown. Elevated cytokines by stress that triggered TS induce tissue and plasma events that lead to changes in lipid metabolism such as hypertriglyceridemia, elevated TGrich lipoproteins, and decreased HDL [12]. Hyperthyroidism has been reported to be characterized by decreases in total cholesterol, LDL-C, as well as HDL-C levels [13]. Severe thyrotoxicosis in TS may strongly lower cytokine-induced elevation of TG. In addition to cytokine, severe thyrotoxicosis may induce further decrease of HDL-C. The remarkable low HDL-C level observed in Graves' disease patients with TS $(29.3 \mathrm{mg} /$ dL) compared to those without TS $(52.9 \mathrm{mg} / \mathrm{dL})$ can be an important characteristic for TS, because such low HDL-C level may reflect severity of thyrotoxicosis and cytokine storm. TS patients showed 23, 25 and $40 \mathrm{mg} / \mathrm{dL}$ of HDL-C, while one patient without TS showed $31 \mathrm{mg} / \mathrm{dL}$ of HDL-C and 48 patients 
Table 5. Characteristics for Graves' Disease Patients With Thyroid Storm Compared to Those Without Thyroid Storm

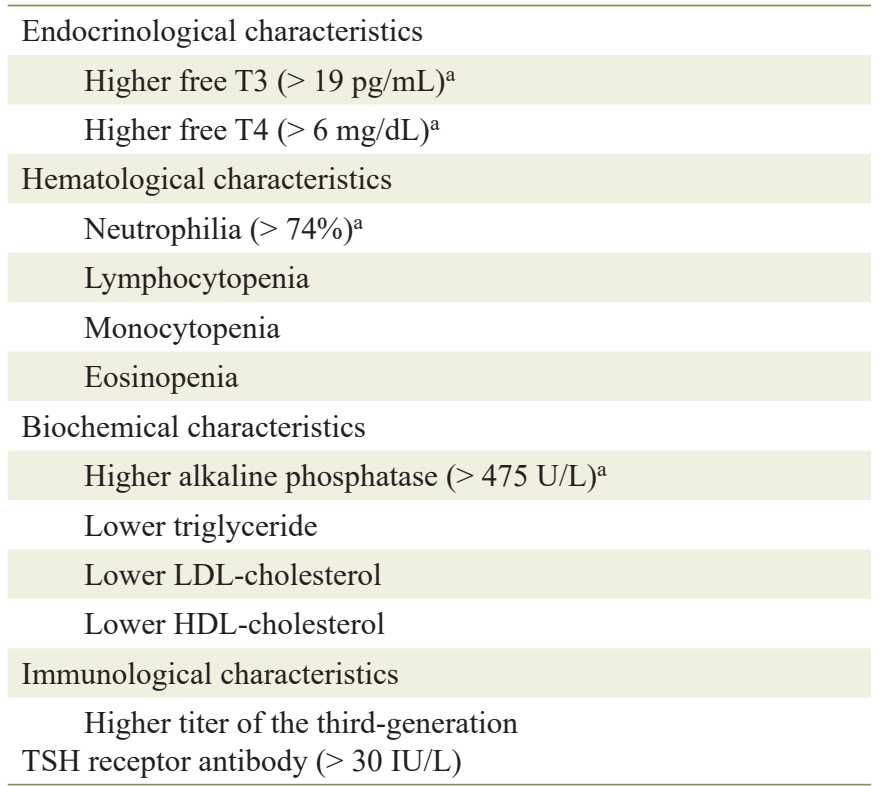

${ }^{a}$ Cutoff values were obtained by a receiver operating characteristics $(R O C)$ curve with area under the curve $(A \cup C)>0.7$.

without TS showed more than $41 \mathrm{mg} / \mathrm{dL}$ of HDL-C, suggesting that HDL-C $\leq 40 \mathrm{mg} / \mathrm{dL}$ may distinguish between patients with and without TS.

In our study, all five patients showed the titer of third-generation TRAb > $30 \mathrm{IU} / \mathrm{L}$, which were significantly higher than those without TS. No patients without TS showed the titer of third-generation TRAb $>30 \mathrm{IU} / \mathrm{L}$, suggesting that the titer of third-generation TRAb $>30 \mathrm{IU} / \mathrm{L}$ has an excellent discriminatory power between patients with and without TS. So far, only positivity of TRAb has been discussed in the pathogenesis of TS; however, the titer of TRAb may be associated with the development of TS. Very recently, we reported a possibility that over-induction of TRAb may trigger the development of TS, by showing two TS cases [14]. Present study is the first to suggest on an association of TRAb titer with TS, which should be confirmed by prospective studies.

Present study has limitations. First, the number of studied subjects was small because of rarity of TS. Second, since this study was retrospective and based on medical charts, lack of data might influence the results. A more detailed prospective study is recommended.

\section{Conclusions}

Characteristics for Graves' disease patients with TS compared to those without TS were shown in Table 5. The titer of thirdgeneration TRAb > 30 IU/L was only observed in Graves' disease patients with TS, but not in Graves' disease patients without TS, suggesting a significance of higher titer of thirdgeneration TRAb ( $>30$ IU/L) for the diagnosis of TS. Furthermore, the cutoff values for fT3, fT4, neutrophil and ALP could be obtained by a receiver operating characteristics (ROC) curve with area under the curve (AUC) $>0.7$.

Our study demonstrated significant clinical, biochemical, hematological, endocrinological and immunological differences in Graves' disease patients with TS compared to those without TS.

\section{Acknowledgments}

We thank the staffs of the Division of Research Support, National Center for Global Health and Medicine Kohnodai Hospital.

\section{Financial Disclosure}

Authors have no financial disclosures to report.

\section{Conflict of Interest}

The authors declare that they have no conflicts of interest concerning this article.

\section{Informed Consent}

Not applicable.

\section{Author Contributions}

H.Y. and M.H. designed the research. M.H. and H.K. collected data. H.Y. analyzed data, and H.Y. wrote the paper. All authors read and approved the final paper.

\section{References}

1. Gavin LA. Thyroid crises. Med Clin North Am. 1991;75(1):179-193.

2. Tietgens ST, Leinung MC. Thyroid storm. Med Clin North Am. 1995;79(1):169-184.

3. Akamizu T, Satoh T, Isozaki O, Suzuki A, Wakino S, Iburi T, Tsuboi K, et al. Diagnostic criteria, clinical features, and incidence of thyroid storm based on nationwide surveys. Thyroid. 2012;22(7):661-679.

4. Isozaki O, Satoh T, Wakino S, Suzuki A, Iburi T, Tsuboi K, Kanamoto N, et al. Treatment and management of thyroid storm: analysis of the nationwide surveys: The taskforce committee of the Japan Thyroid Association and Japan Endocrine Society for the establishment of diagnostic criteria and nationwide surveys for thyroid storm. Clin Endocrinol (Oxf). 2016;84(6):912-918.

5. Nai Q, Ansari M, Pak S, Tian Y, Amzad-Hossain M, Zhang Y, Lou Y, et al. Cardiorespiratory Failure in Thy- 
roid Storm: Case Report and Literature Review. J Clin Med Res. 2018;10(4):351-357.

6. Akamizu T. Thyroid Storm: A Japanese perspective. Thyroid. 2018;28(1):32-40.

7. Satoh T, Isozaki O, Suzuki A, Wakino S, Iburi T, Tsuboi K, Kanamoto N, et al. 2016 Guidelines for the management of thyroid storm from The Japan Thyroid Association and Japan Endocrine Society (First edition). Endocr J. 2016;63(12):1025-1064.

8. Riad M, Mogos M, Thangathurai D, Lumb PD. Steroids. Curr Opin Crit Care. 2002;8(4):281-284.

9. Fauci AS, Dale DC, Balow JE. Glucocorticosteroid therapy: mechanisms of action and clinical considerations. Ann Intern Med. 1976;84(3):304-315.

10. Al-Nuaim A, El-Desouki M, Sulimani R, Mohammadiah $\mathrm{M}$. Bone mineral density and metabolic indices in hyper- thyroidism. Ann Saudi Med. 1991;11(5):530-533.

11. Cooper DS, Kaplan MM, Ridgway EC, Maloof F, Daniels GH. Alkaline phosphatase isoenzyme patterns in hyperthyroidism. Ann Intern Med. 1979;90(2):164-168.

12. Khovidhunkit W, Memon RA, Feingold KR, Grunfeld C. Infection and inflammation-induced proatherogenic changes of lipoproteins. J Infect Dis. 2000;181(Suppl 3):S462-472.

13. Feingold K, Brinton EA, Grunfeld C. The effect of endocrine disorders on lipids and lipoproteins. Endotext [Internet]. South Dartmouth (MA): MDText.com, Inc.; 2000-22017 Feb 24.

14. Hakoshima M, Yanai H, Komatsuzaki S, Kawaguchi A, Hamasaki H, Katsuyama H. Streptococcal infection can be the trigger for thyroid storm. J Clin Med Res. 2019;11(5):383-384. 